\title{
One Dimensional Conservative System with Quadratic Dissipation and Position Depending Mass
}

\author{
Gustavo V. López, Erick S. Madrigal \\ Departamento de Física, Universidad de Guadalajara, Blvd. Marcelino García Barragan y Calzada Olímpica, Guadalajara, Jalisco, \\ Mexico \\ Email: gulopez@cencar.udg.mx, erick.madrigal8089@alumnos.udg.mx
}

How to cite this paper: López, G.V. and Madrigal, E.S. (2022) One Dimensional Conservative System with Quadratic Dissipation and Position Depending Mass. Journal of Modern Physics, 13, 127-137. https://doi.org/10.4236/jmp.2022.132011

Received: January 1, 2022

Accepted: February 11, 2022

Published: February 14, 2022

Copyright (๑) 2022 by author(s) and Scientific Research Publishing Inc. This work is licensed under the Creative Commons Attribution International License (CC BY 4.0).

http://creativecommons.org/licenses/by/4.0/

\begin{abstract}
Forl a 1-D conservative system with a position depending mass within a dissipative medium, its effect on the body is to exert a force depending on the squared of its velocity, a constant of motion, Lagrangian, generalized linear momentum, and Hamiltonian are obtained. We apply these new results to the harmonic oscillator and pendulum under the characteristics mentioned about, obtaining their constant of motion, Lagrangian and Hamiltonian for the case when the body is increasing its mass.
\end{abstract}

\section{Keywords}

Dissipation, Position Mass Depending, Constant of Motion, Hamiltonian

\section{Introduction}

Variable mass problems without dissipation have a long history and are known as Gylden-Meshcherskii problems [1] [2] [3] [4] [5]. As it is known, Newton's equation with position mass depending is not invariant under Galileo's transformation [6] [7], and Sommerfeld gave a modification of this equation to overcome this problem [8]. However, this modification has a fundamental problem when external force is zero, and that is why one considers Newton's equation of motion as a good equation of motion for these types of problems [9] [10]. This approach was used for 1-D conservative systems with position depending mass [11], binary stars with mass exchanged [12] [13], binary galaxies with mass exchanged [14], and fluid dynamics [15]. On the other hand, 1-D systems with constant mass and quadratic dissipation have also been studied [16]. Therefore, in this paper both situations are considered at the same time, position mass de- 
pending and quadratic dissipation on 1-D conservative systems, and for these systems one will find a Constant of Motion, Lagrangian, Generalized Linear Momentum, and Hamiltonian. The results will be applied to the study on the dynamics of the harmonic oscillator and pendulum systems with this dissipation and with increasing of mass behavior.

\section{Analytical Approach}

Newton's equation for 1-D conservative systems, characterized by an external force $F(x)$, with position depending mass, $m(x)$, and a quadratic dissipation force, $-\alpha v^{2}$ (being $\alpha$ a nonnegative real constant, and $v \geq 0$ ), is given by

$$
\frac{\mathrm{d}}{\mathrm{d} t}(m(x) v)=F(x)-\alpha v^{2}, \quad v \geq 0,
$$

where $v$ represents the velocity, $v=\mathrm{d} x / \mathrm{d} t=\dot{x}$, of the body, and $\alpha$ is a constant. One will consider that $m(0)=m_{o}$ represents the initial mass of the system at the point $x=0$. Equation (1) can be written as an autonomous dynamical system defined in $\mathfrak{R}^{2}$ as

$$
\begin{gathered}
\dot{x}=v, \\
\dot{v}=\frac{F(x)-\left(\alpha+m_{x}\right) v^{2}}{m(x)},
\end{gathered}
$$

where $m_{x}$ has been defined as $m_{x}=\mathrm{d} m / \mathrm{d} x$. A constant of motion for this system is a function $K=K(x, v)$ such that $\mathrm{d} K / \mathrm{d} t=0$, that is, it must satisfy the following first order partial differential equation

$$
v \frac{\partial K}{\partial x}+\frac{F(x)-\left(\alpha+m_{x}\right) v^{2}}{m(x)} \frac{\partial K}{\partial v}=0
$$

which can be solved by the characteristics method [17], where the equations for the characteristics are

$$
\frac{\mathrm{d} x}{v}=\frac{m(x) \mathrm{d} v}{F(x)-\left(\alpha+m_{x}\right) v^{2}}=\frac{\mathrm{d} K}{0} .
$$

The last term just tell us that the function $K$ must be an arbitrary function of the characteristic $C$ obtained from the others two terms, $K=G(C)$ where $G$ is arbitrary. From the others two terms, one can write the following equation

$$
\frac{m(x)}{2} \frac{\mathrm{d} v^{2}}{\mathrm{~d} x}+\left(\alpha+m_{x}\right) v^{2}=F(x) .
$$

Defining a new variable $\xi$ as $\xi=v^{2}$ and rearranging terms, this equation is written as

$$
\frac{\mathrm{d} \xi}{\mathrm{d} x}+\frac{2\left(\alpha+m_{x}\right)}{m(x)} \xi=\frac{2 F(x)}{m(x)} .
$$

Now, multiplying this equation by $\exp \left(\int^{x} \frac{2\left(\alpha+m_{x}\right)}{m(s)} \mathrm{d} s\right)$, the resulting equa- 
tion can be written as

$$
\frac{\mathrm{d}}{\mathrm{d} x}\left(\xi \mathrm{e}^{\int^{x} \frac{2\left(\alpha+m_{x}\right) \mathrm{d} s}{m(s)}}\right)=\frac{2 F(x)}{m(x)} \mathrm{e}^{\int^{x} \frac{2\left(\alpha+m_{x}\right) \mathrm{d} s}{m(s)}},
$$

which can easily be integrated, and one gets the following expression in terms of the variable $V$

$$
v^{2} \mathrm{e}^{\int^{x} \frac{2\left(\alpha+m_{x}\right) d s}{m(s)}}=2 \int \frac{F(x)}{m(x)} \mathrm{e}^{\int^{x} \frac{2\left(\alpha+m_{x}\right) d s}{m(s)}} \mathrm{d} x+A,
$$

where $A$ is the constant of integration. Then, one chooses the characteristic curve as $C=m_{o} A / A$ and chooses the function $G$ as $G(C)=C$ to get the constant of motion

$$
K_{\alpha}^{(+)}(x, v)=\frac{m_{o} v^{2}}{2} \mathrm{e}^{\int^{x} \frac{2\left(\alpha+m_{x}\right) \mathrm{d} s}{m(s)}}-m_{o} \int \frac{F(x)}{m(x)} \mathrm{e}^{\int^{x} \frac{2\left(\alpha+m_{x}\right) d s}{m(s)}} \mathrm{d} x .
$$

Using the following identity

$$
\mathrm{e}^{\int^{x} \frac{2 m_{x}(s) \mathrm{d} s}{m(s)}}=\left(\frac{m(x)}{m_{o}}\right)^{2}
$$

the expression (9a) is written finally as

$$
K_{\alpha}^{(+)}(x, v)=\frac{m^{2}(x) v^{2}}{2 m_{o}} \mathrm{e}^{2 \alpha \int^{x} \frac{\mathrm{d} s}{m(s)}}-\frac{1}{m_{o}} \int^{x} m(x) F(x) \mathrm{e}^{2 \alpha \int^{x} \frac{\mathrm{d} s}{m(s)}} \mathrm{d} x .
$$

This expression is of the form

$$
K_{\alpha}^{(+)}(x, v)=T_{\alpha}(x, v)+V_{\alpha}(x)
$$

where $T_{\alpha}$ is some type of effective kinetic energy of the system,

$$
T_{\alpha}(x, v)=\frac{m^{2}(x) v^{2}}{2 m_{o}} \mathrm{e}^{2 \alpha \int^{x} \frac{\mathrm{d} s}{m(s)}}
$$

and $V_{\alpha}$ is just the effective potential

$$
V_{\alpha}(x)=-\frac{1}{m_{o}} \int^{x} m(x) F(x) \mathrm{e}^{2 \alpha \int^{x} \frac{\mathrm{d} s}{m(s)}} \mathrm{d} x .
$$

Then, one can say that $K_{\alpha}^{(+)}$represents the effective energy of the system.

\subsection{Special Cases}

Let us note the following:

First, one has the following limit

$$
\lim _{\alpha \rightarrow 0} K_{\alpha}^{(+)}(x, v)=\frac{m^{2}(x) v^{2}}{2 m_{o}}-\frac{1}{m_{o}} \int^{x} m(x) F(x) \mathrm{d} x,
$$

which is the expression obtained in reference [11].

Second, assuming the mass as constant, $m(x)=m_{o}$, one gets 


$$
K_{\alpha}^{(+)}(x, v)=\frac{m_{o} v^{2}}{2} \mathrm{e}^{2 \alpha x / m_{o}}-\int^{x} F(x) \mathrm{e}^{2 \alpha x / m_{o}} \mathrm{~d} x,
$$

which is the expression obtained in references [16] [18] (for the non relativistic case).

Third, for $\alpha=0$ and $m(x)=m_{o}$, one gets the usual energy of a conservative system

$$
K(x, v)=\frac{m_{o}}{2} v^{2}+V(x),
$$

where $V(x)$ is the potential of the system, $V(x)=-\int F(x) \mathrm{d} x$.

\subsection{Lagrangian and Hamiltonian}

Now, since $K_{\alpha}^{(+)}(x, v)$ is a constant of motion, a Lagrangian of the system can be found through the relation [19] [20] [21]

$$
L_{\alpha}^{(+)}(x, v)=v \int \frac{K_{\alpha}^{(+)}(x, \xi) \mathrm{d} \xi}{\xi^{2}} .
$$

In this way and considering (13), one gets

$$
L_{\alpha}^{(+)}(x, v)=\frac{m^{2}(x) v^{2}}{2 m_{o}} \mathrm{e}^{2 \alpha \int^{x} \frac{\mathrm{d} s}{m(s)}}-V_{\alpha}(x)
$$

The generalized linear momentum is

$$
p_{\alpha}^{(+)}(x, v)=\frac{m^{2}(x) v}{m_{o}} \mathrm{e}^{2 \alpha \int^{x} \frac{\mathrm{d} s}{m(s)}} .
$$

With this expression and the Legendre's transformation, $H(x, p)=v(x, p) p-L(x, v(x, p))$, the Hamiltonian of the system is given by

$$
H_{\alpha}^{(+)}(x, p)=\frac{m_{o} p^{2}}{2 m(x)} \mathrm{e}^{-2 \alpha \int^{x} \frac{\mathrm{d} s}{m(s)}}+V_{\alpha}(x)
$$

If we apply the above observations (11) on the expressions (10), (15), (16), and (17), one gets the corresponding correct expression for these cases.

Let us notice from (1) that the dissipation for $v<0$ can be obtained by making the change $\alpha \rightarrow-\alpha$ on the expressions already found. Therefore, the constant of motion, Lagrangian, generalized linear momentum, and Hamiltonian when $v<0$ are given by

$$
\begin{gathered}
K_{\alpha}^{(-)}(x, v)=K_{-\alpha}^{(+)}(x, v), \\
L_{\alpha}^{(-)}(x, v)=L_{-\alpha}^{(+)}(x, v), \\
p_{\alpha}^{(-)}(x, v)=p_{-\alpha}^{(+)}(x, v),
\end{gathered}
$$

and

$$
H_{\alpha}^{(-)}(x, p)=H_{-\alpha}^{(+)}(x, p) .
$$

However, notice from (13) that the potential $V_{-\alpha}(x)$ can be very different 
from $V_{\alpha}(x)$, as it will be seen on below examples.

\section{Mass Linear Dependence on Position}

In this case, one has the following dependence of the mass with respect the position of the body

$$
m(x)=m_{o}+\beta x,
$$

where $\beta$ is a constant. Then, it follows that

$$
\mathrm{e}^{2 \alpha \int^{x} \frac{\mathrm{ds}}{m(s)}}=\left(\frac{m_{o}+\beta x}{m_{o}}\right)^{2 \alpha / \beta} .
$$

So, form the expressions (10), (15), (16), and (17), one obtains

$$
\begin{gathered}
K_{\alpha}^{(+)}(x, v)=\frac{\left(m_{o}+\beta x\right)^{2+2 \alpha / \beta}}{2 m_{o}^{1+2 \alpha / \beta}} v^{2}+V_{\alpha}(x), \\
L_{\alpha}^{(+)}(x, v)=\frac{\left(m_{o}+\beta x\right)^{2+2 \alpha / \beta}}{2 m_{o}^{1+2 \alpha / \beta}} v^{2}-V_{\alpha}(x), \\
p_{\alpha}^{(+)}(x, v)=\frac{\left(m_{o}+\beta x\right)^{2+2 \alpha / \beta}}{m_{o}^{1+2 \alpha / \beta}} v,
\end{gathered}
$$

and

$$
H_{\alpha}^{(+)}(x, p)=\frac{m_{o}^{1+2 \alpha / \beta}}{2\left(m_{o}+\beta x\right)^{2+2 \alpha / \beta}} p^{2}+V_{\alpha}(x),
$$

where the effective potential $V_{\alpha}$ is given by

$$
V_{\alpha}(x)=-\frac{1}{m_{o}^{1+2 \alpha / \beta}} \int^{x} F(x)\left(m_{o}+\beta x\right)^{1+2 \alpha / \beta} \mathrm{d} x .
$$

\subsection{Harmonic Oscillator}

For the harmonic oscillator, one has that $F(x)=-k x$, and using the following integration

$$
\int x\left(m_{o}+\beta x\right)^{1+2 \alpha / \beta} \mathrm{d} x=\frac{1}{\beta^{2}}\left(m_{o}+\beta x\right)^{2+2 \alpha / \beta}\left(2 \beta x+2 \alpha x-m_{o}\right),
$$

the effective potential is

$$
V_{\alpha}(x)=\frac{k\left(m_{o}+\beta x\right)^{2+2 \alpha / \beta}\left(2 \beta x+2 \alpha x-m_{o}\right)}{\beta^{2} m_{o}^{1+2 \alpha / \beta}(3+2 \alpha / \beta)(2+2 \alpha / \beta)},
$$

where the constant term $-k m_{o}^{2}\left(1 / 4 \alpha^{2}+1 / 6 \beta^{2}\right)$ could be added to get the right limits $\left(\lim _{\alpha \rightarrow 0} \lim _{\beta \rightarrow 0} V_{\alpha}=\lim _{\beta \rightarrow 0} \lim _{\alpha \rightarrow 0} V_{\alpha}=k x^{2} / 2\right)$, and one obtains the constant of motion, Lagrangian, generalized linear momentum, and Hamiltonian for the harmonic oscillator with linear position dependence on its mass and quadratic dissipation as

$$
K_{\alpha}^{(+)}(x, v)=\frac{\left(m_{o}+\beta x\right)^{2+2 \alpha / \beta}}{2 m_{o}^{1+2 \alpha / \beta}} v^{2}+V_{\alpha}(x)
$$




$$
\begin{gathered}
L_{\alpha}^{(+)}(x, v)=\frac{\left(m_{o}+\beta x\right)^{2+2 \alpha / \beta}}{2 m_{o}^{1+2 \alpha / \beta}} v^{2}-V_{\alpha}(x) \\
p_{\alpha}^{(+)}(x, v)=\frac{\left(m_{o}+\beta x\right)^{2+2 \alpha / \beta}}{m_{o}^{1+2 \alpha / \beta}} v,
\end{gathered}
$$

and

$$
H_{\alpha}^{(+)}(x, p)=\frac{m_{o}^{1+2 \alpha / \beta} p^{2}}{2\left(m_{o}+\beta x\right)^{2+2 \alpha / \beta}}+V_{\alpha}(x) .
$$

Figure 1 shows the behavior of the body in the one quarter of the phase spaces $(x, v)$ and $(x, p)$ for several values of the parameter $K$, with $m_{o}=1 \mathrm{~kg}$,

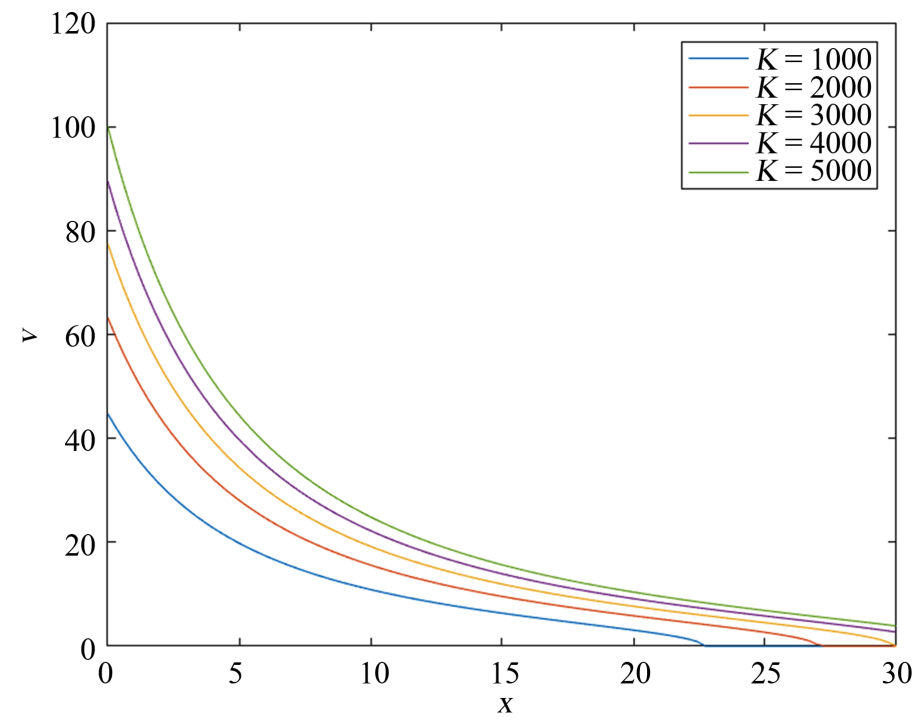

(a)

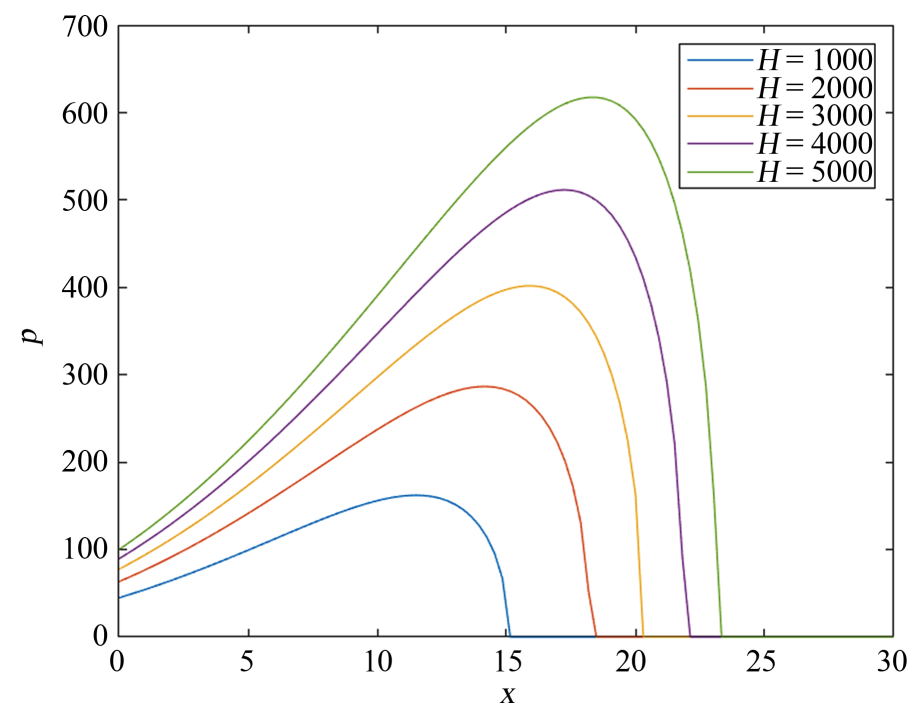

(b)

Figure 1. Behavior through the constant of motion and the hamiltonian. (a) $\alpha=\beta=0.1 \mathrm{~kg} / \mathrm{m}$; (b) $\alpha=\beta=0.1 \mathrm{~kg} / \mathrm{m}$. 
and $k=1 \mathrm{~N} / \mathrm{m}$. Note that since $\beta \geq 0$, the system is acquiring mass as the position is increasing. Because of this, and due that one has dissipation in the system, the body will perform a damping spiral behavior on the phase spaces $(x, v)$ and $(x, p)$, which is not shown here.

To determine this spiral damping behavior and assuming always and increasing of mass, one would have to divide the phase space $(x, v)$ in four regions: 1$)$ $v>0$ and $x>0,2) \quad v<0$ and $s \geq 0,3) \quad v<0$ and $s<0,4) \quad v>0$ and $s \leq 0$. On the upper place $(v>0)$ one uses $K_{\alpha}^{(+)}$, and in the lower plane one uses $K_{\alpha}^{(-)}$. Once $v=0$ on the region (1), the effective energy $K_{\alpha}^{(-)}$is determine by the value of the effective potential at the point $x_{1}$ where this happens, and the mass changes on the region (2) of the form $m(s)=m_{o}+\beta s_{1}+\beta\left(s_{1}-s\right)$ in the interval $s \in\left[s_{1}, 0\right]$. On the region (3) the mass must vary as $m(s)=m_{o}+2 \beta s_{1}+\beta|s|$ until the body reaches again a velocity $v=0$ at the point $s_{2}$ (negative). At this point the effective energy $K_{\alpha}^{(+)}$is defined by the value of the effective potential at this point, and the mass varies on this region (4) as $m(s)=m_{o}+2 \beta s_{1}+\beta\left|s_{2}\right|+\beta\left(\left|s_{2}\right|-|s|\right)$ until the body reaches $s=0$, completing on cycle of the spiral motion. The same would be repeated with the other cycles of the spiral motion. The reason of this complication is due to the fact that during the whole motion the body is increasing its mass, otherwise one would have mass oscillation depending whether $x$ is positive or negative. The same idea is applied for the Hamiltonian and the phase space $(x, p)$, and note the great different behavior of body on the phase space $(x, p)$ with respect the phase behavior on the phase space $(x, v)$, due to the position dependence of the generalized linear momentum $(24 \mathrm{c})$.

\subsection{Pendulum}

The position on the pendulum is determined by its displacement $s$ respect its equilibrium position at the angle $\theta=0$, that is $s=l \theta$, where $l$ denotes the length of the cord. The force acting on the body, of mass $m(s)$, hanged at the end of the cord is given by $F(s)=-g m(s) \sin (s / l)$, being $g$ the constant acceleration due to gravity. Using the following integration [22]

$$
\begin{aligned}
& f(s)=\int F(s)(1+\beta s)^{1+2 \alpha / \beta} \mathrm{d} s=-\frac{g(\beta l)^{3+2 \alpha / \beta}}{2 \beta} \mathrm{e}^{i\left(m_{o} / \beta l-\pi \alpha / \beta\right)} \\
& \times\left\{\gamma\left(3+\frac{2 \alpha}{\beta},-i \frac{m_{o}+\beta s}{\beta l}\right)+(-1)^{3+2 \alpha / \beta} \gamma\left(3+\frac{2 \alpha}{\beta}, i \frac{m_{o}+\beta s}{\beta l}\right)\right\},
\end{aligned}
$$

where $\gamma$ is the uncompleted gamma function [22] (page 940). If we select the mass as $m_{o}=1 \mathrm{~kg}$ and $\alpha / \beta=n$ and integer number, the function $f(s)$ can be given by

$$
f(s)=-g \sum_{k=0}^{2+n} \frac{(2+n) !(\beta l)^{k}}{(2+n-k) !} \sum_{j=0}^{k} \frac{s^{k-j}}{l^{k-j}(k-j) !} \cos (s / l+j \pi / 2) .
$$

Therefore, the effective potential is 


$$
V_{\alpha}(s)=\frac{f(s)}{m_{o}^{1+2 \alpha / \beta}} .
$$

The Constant of Motion, Lagrangian, generalized linear momentum, and Hamiltonian are

$$
\begin{gathered}
K_{\alpha}^{(+)}(s, v)=\frac{\left(m_{o}+\beta s\right)^{2+2 \alpha / \beta}}{2 m_{o}^{1+2 \alpha / \beta}} v^{2}+V_{\alpha}(s) \\
L_{\alpha}^{(+)}(s, v)=\frac{\left(m_{o}+\beta s\right)^{2+2 \alpha / \beta}}{2 m_{o}^{1+2 \alpha / \beta}} v^{2}-V_{\alpha}(s) \\
p_{\alpha}^{(+)}(s, v)=\frac{\left(m_{o}+\beta s\right)^{2+2 \alpha / \beta}}{m_{o}^{1+2 \alpha / \beta}} v,
\end{gathered}
$$

and

$$
H_{\alpha}^{(+)}(s, p)=\frac{m_{o}^{1+2 \alpha / \beta}}{2\left(m_{o}+\beta s\right)^{2+2 \alpha / \beta}} p^{2}+V_{\alpha}(s),
$$

where $v$ represents the velocity of the body, $v=\mathrm{d} s / \mathrm{d} t$. Figure 2 shows the behavior of the body in the first quadrant $(s \geq 0, v \geq 0$, and $p \geq 0)$ of the phase spaces $(s, v)$ and $(s, p)$ for the values of the parameters $K$ and $H$ given by $0,10,20,30,40,50,60,70$, and 80 (blue, orange, yellow, and so on) with $m_{o}=1 \mathrm{~kg}$, and $l=1 \mathrm{~m}$ and $f(s)$ taken as the expression (25b). The inner blue and orange lines represent the oscillatory spiral damping behavior of the body

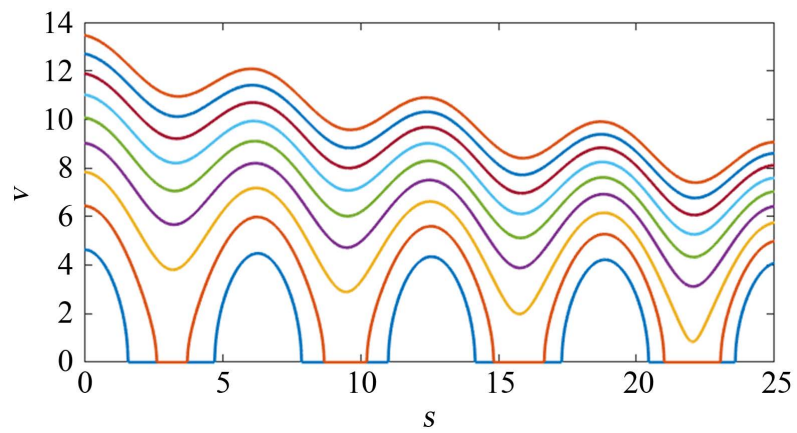

(a)

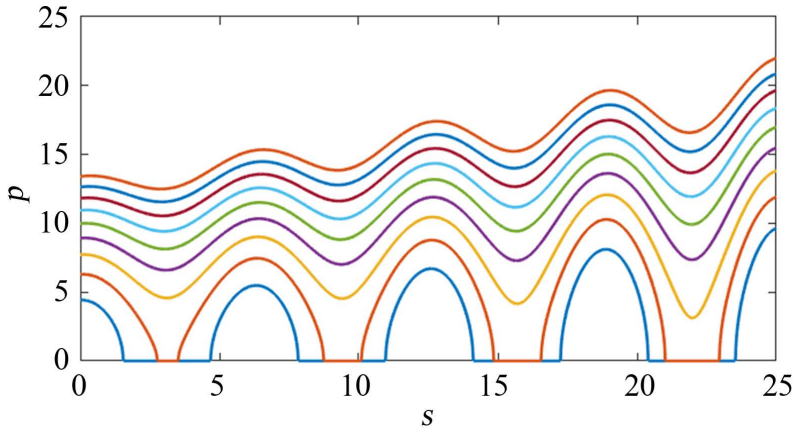

(b)

Figure 2. Behavior through the constant of motion and the hamiltonian. (a) Phase space $(x, v)$; (b) Phase space $(x, p)$. 
due to increasing of mass during its motion and the damping factor. The upper lines represent the rotational spiral damping behavior of the body due to the same reason (this spiral damping behavior is not shown on these plots. To get this behavior one would need to proceed similarly as it was explained for the harmonic oscillator part).

The effective potential $V_{\alpha}$ has an oscillatory increasing behavior as a function of the displacement $s$. Therefore, it does not matter which value of the effective energy $K$ or $H$ takes, due to the increasing of mass and damping factor, the body will perform an oscillatory damping behavior, that is, the origin of the phase space is an attractor of the dynamics of the body (as it happened with the first example). On Figure 2(b) one sees an apparent increasing of the generalized linear momentum as the body is rotating. However, eventually will reach the return point of the potential and the generalized linear momentum will be zero (as the yellow line indicates).

\section{Conclusions and Comments}

In general, we have constructed constant of motion, Lagrangian, generalized linear momentum, and Hamiltonian for a 1-D conservative system with position depending mass and embedded in a medium where the body feels a dissipative force which depends quadratically on its velocity. In particular, we made the analysis for the case when the body increases its mass linearly on its displacement, where the dynamics in the phase spaces $(x, v)$ and $(x, p)$ is plotted on one quadrant of these spaces, which could be very important if one wants to use quantum mechanics for theses system, and we have shown the damping effect on the motion of the body for the harmonic oscillator and pendulum systems due to dissipative force and the increasing of its mass.

We want to comment something for the case of mass lost, we have seen from our model (19) with $\beta<0$ that the motion is limited to a displacement given by $x_{\max }=m_{o} /|\beta|$ (zero mass), where the potential function of the harmonic oscillator is zero, and this value would represent a singularity in the velocity behavior for positive generalized energies (for generalized energies higher than $\left.V_{\alpha}\left(x_{\max }\right)\right)$, but it would represent a zero motion in space $(x, p)$ for the Hamiltonian.

\section{Conflicts of Interest}

The authors declare no conflicts of interest regarding the publication of this paper.

\section{References}

[1] Gylden, H. (1884) Astronomishe Nachrichten, 109, 1-6.

[2] Meshcherskii, I.V. (1983) Astronomishe Nachrichten, 132, 1-9.

[3] Meshcherskii, I.V. (1902) Astronomishe Nachrichten, 159, 229-242. https://doi.org/10.1002/asna.19021591502 
[4] Prieto, C. and Docobo, J.A. (1997) Celestial Mechanics and Dynamical Astronomy, 68, 53-62. https://doi.org/10.1023/A:1008235630740

[5] Cveticanin, L. (2012) Journal of the Serbian Society for Computational Mechanics, 6, 56-73.

[6] Goldstein, H. (1950) Classical Mechanics. Addison-Wesley, Boston.

[7] Arnold, V.I. (1989) Mathematical Methods of Classical Mechanics. Springer-Verlag, Berlin. https://doi.org/10.1007/978-1-4757-2063-1

[8] Sommerfeld, A. (1964) Lectures on Theoretical Physics, Volume 1. Academic Press, Cambridge.

[9] Spivak, M. (2010) Physics for Mathematicians, Mechanics I, Volume 1. Publish or Perish, Inc., Houston, TX.

[10] López, G.V. and Juárez, E.M. (2013) Journal of Modern Physics, 4, 1638-1649. https://doi.org/10.4236/jmp.2013.412204

[11] López, G.V. and Prieto, C.R.M. (2014) Journal of Modern Physics, 5, 900-907.

[12] López, G. and López, E. (2018) Journal of Applied Mathematical and Physics, 6, 613-619. https://doi.org/10.4236/jamp.2018.63053

[13] Hamilton, C. and Rafikov, R.R. (2019) Monthly Notices of the Royal Astronomical Society, 488, 5512-5535. https://doi.org/10.1093/mnras/stz2026

[14] López, E.L., López, G.V. and Kemp, S.N. (2018) The Astronomical Journal, 156, 215. https://doi.org/10.3847/1538-3881/aae08c

[15] Nyabuto, R., Amenya, R. and Okelo, J. (2021) Journal of Applied Mathematic and Physics, 9, 931-940. https://doi.org/10.4236/jamp.2021.95063

[16] López, G.V., Montes, G.C. and Zanudo, J.G.T. (2015) Journal of Modern Physics, 6, 121-125. https://doi.org/10.4236/jmp.2015.62016

[17] John, F. (1974) Partial Differential Equations, Volume 1. Spring-Verlag, Berlin.

[18] López, G.V. (2012) Journal of Modern Physics, 3, 777-785. https://doi.org/10.4236/jmp.2012.38102

[19] Kobussen, J.A. (1979) Acta Physica Austriaca, 51, 293-309.

[20] Leubner, C. (1981) Physica A, 86, 68. https://doi.org/10.1016/0375-9601(81)90166-3

[21] López, G.V. (1996) Annals of Physics, 251, 372-383. https://doi.org/10.1006/aphy.1996.0118

[22] Gradsteyn, I.S. and Ryzhik, I.M. (1980) Table of Integrals, Series, and Products. Academic Press, Cambridge. 


\section{List of Terminology}

$\alpha:$ Dissipation parameter;

$\beta$ : Variation of mass with respect to " $X$ " parameter;

$F(x)$ : Conservative force;

$K_{\alpha}^{ \pm}(x, v)$ : Constant of motion $(+(v>0)$ and $-(v>0))$ or Effective energy;

$T_{\alpha}(x, v)$ : Effective Kinetic Energy;

$V_{\alpha}(x)$ : Effective Potential;

$L_{\alpha}^{ \pm}(x, v)$ : Lagrangian $(+(v>0)$ and $-(v<0))$;

$p_{\alpha}^{ \pm}(x, v)$ : Generalized Linear Momentum $(+(v>0)$ and $-(v<0))$;

$H_{\alpha}^{ \pm}(x, p)$ : Hamiltonian $(+(p>0)$ and $-(p<0))$;

$\gamma(a, b)$ : Uncompleted gamma function. 